\title{
Electron-Proton Instability in the IPNS-Upgrade RCS: Part I
}

\author{
Yong-Chul Chae \\ Accelerator Systems Division \\ Argonne National Laboratory \\ 9700 S. Cass Ave., Argonne, IL 60439 \\ February 27, 1995
}

\begin{abstract}
The electron-proton instability in the IPNS-Upgrade RCS is investigated in this report. A dispersion relation applicable to the coasting beam is derived, and the approximations used are stated in order to facilitate the understanding of the underlying mechanism of the instability. The threshold of instability in terms of neutralization of the circulating beam is found for the IPNS-Upgrade RCS. The dependence of threshold on the beam size and the lattice tune is also studied and its numerical results are presented.
\end{abstract}




\section{DISCLAIMER}

This report was prepared as an account of work sponsored by an agency of the United States Government. Neither the United States Government nor any agency thereof, nor any of their employees, make any warranty, express or implied, or assumes any legal liability or responsibility for the accuracy, completeness, or usefulness of any information, apparatus, product, or process disclosed, or represents that its use would not infringe privately owned rights. Reference herein to any specific commercial product, process, or service by trade name, trademark, manufacturer, or otherwise does not necessarily constitute or imply its endorsement, recommendation, or favoring by the United States Government or any agency thereof. The views and opinions of authors expressed herein do not necessarily state or reflect those of the United States Government or any agency thereof. 


\section{DISCLAIMER}

Portions of this document may be illegible in electronic image products. Images are produced from the best available original document. 


\section{Introduction}

The residual gas in the vacuum chamber is ionized by the circulating beam due to the collisional processes. The electrons liberated from the molecules can be trapped in the proton beam's potential well. Since the trapped electrons oscillate in the potential well, the transverse motion of electrons and protons are coupled, which can result in amplitude growth. This instability was first observed in the coasting beam at the CERN-ISR and has been called electron-proton ("e-p") instability [1] since then. This instability is characterized by a growth time which is comparable to the fastest instabilities caused by coupling impedances. This instability should not be a concern in the IPNS-Upgrade RCS because the circulating beam is bunched, and it has not been observed in similar rapid cycling machines, including ISIS [2], KENS, and IPNS.

However, a fast instability, known as the "PSR instability" and similar to the e-p instability, was recently observed at PSR at Los Alamos during chopped beam accumulation [3]. Whether or not this is a true e-p instability is unknown, however prior to the onset of the instability, the gap between the bunch passages is filled by bunch leakage. This is consistent with e-p type instability.

In the IPNS-Upgrade RCS, RF voltage is high enough to avoid bunch leakage during the acceleration period. However, the longitudinal beam distribution in the waiting bucket during injection is sensitive to both chopper efficiency and injection mismatch from the linac. Thus in this report we investigate the possibility of e-p instability during injection assuming that electrons are trapped.

In the following sections, we derive and apply to the IPNS-Upgrade RCS the stability criterion based on the coasting beam theory ${ }^{1}$.

\section{Equations of Motion}

We consider a coasting beam with an elliptic cross section with half-axes $a$ and $b$. We assume the beam has a uniform density $n$, where $n$ is the number of particles per unit volume. This beam represents line density $\lambda=\pi a b n$ with the total current $I=e \lambda \beta c$. The electric and magnetic fields inside such a beam are given by [7]

$$
\mathbf{E}=\frac{e \lambda}{\pi \epsilon_{0}(a+b)}\left(\frac{x}{a}, \frac{y}{b}, 0\right), \quad \mathbf{B}=\frac{\mu_{0} e \lambda}{\pi(a+b)}\left(-\frac{y}{b}, \frac{x}{a}, 0\right) .
$$

\footnotetext{
${ }^{1}$ Such criterion with varying degrees of sophistication can be found in articles written by Baconnier [4], Koshkarov and Zenkevich [5], and Laslett and Sessler [6].
} 
The space-charge force acting on a test particle with charge $e^{*}$ moving with the longitudinal velocity $\beta^{*}=v^{*} c$ is

$$
\mathbf{F}=e^{*}\left(\mathbf{E}+\mathbf{v}^{*} \times \mathbf{B}\right)=\frac{e e^{*} \lambda}{\pi \epsilon_{0}(a+b)\left(1-\beta \beta^{*}\right)}\left(\frac{x}{a}, \frac{y}{b}, 0\right) .
$$

We note that, if the test particle and the beam are of the same charge, the spacecharge force is repulsive; otherwise the force is attractive. For the (square-pulse) bunched beam, we substitute $\lambda$ with $\lambda / B$, where $B$ is the bunching factor defined as bunch-length/circumference.

Since in the RCS the circulating beam is proton, ions will be repelled from the beam and electrons will be attracted toward the beam. The expression for the potential well inside the beam becomes

$$
V(r)=-\int_{0}^{r} E_{r}\left(r^{\prime}\right) d r^{\prime}+V_{0}[\text { Volts }]
$$

where $V_{0}$ fixes the reference potential. We choose $V_{0}$ such that the potential at the edge of the beam is equal to zero. Thus $V_{0}$ represents the potential well depth inside the beam which, for the uniform beam with the radius $a$, is given by

$$
V_{0}=\frac{e \lambda / B}{4 \pi \epsilon_{0}}
$$

The potential difference between the beam edge and the vacuum chamber wall can be written similarly as

$$
\Delta V=2 \ln \frac{r_{0}}{a} V_{0},
$$

where $r_{0}$ is the chamber radius. If the transverse kinetic energy of electrons produced at the beam edge is less than $\Delta V$, all electrons produced within the beam will be trapped. However, since $\Delta V$ is comparable to or larger than $V_{0}$ in most of the accelerators, a convenient trapping condition is that the kinetic energy of electrons is less than $V_{0}$.

By using the following RCS parameters

$$
\begin{aligned}
N_{p}(\text { number of proton in the beam) } & =1.0 \times 10^{14}, \\
<a>(\text { average radius of beam }) & =5.0 \mathrm{~cm}, \\
R \text { (average radius of ring) } & =30.6 \mathrm{~m}, \\
\gamma(\text { injection energy, } 400 \mathrm{MeV}) & =1.4263, \\
\beta(\text { injection energy, } 400 \mathrm{MeV}) & =0.713,
\end{aligned}
$$

we find $V_{0}=760 / B$ Volts. Thus, the electrons with average kinetic energy $10-20 \mathrm{eV}$ [8] will be trapped. We note that if the bunching factor is 0.75 , less than $1 \%$ of beam 
leaked into the bunch gap is enough to trap the electrons with kinetic energy of 20 $\mathrm{eV}$.

The motion of the particle (proton) in the circulating beam in the absence of space-charge force is described by the equation

$$
\frac{d^{2} y_{p}}{d t^{2}}+\nu_{0 y}^{2} \omega_{0}^{2} y_{p}=0
$$

where $\nu_{0 y}$ is the tune of betatron oscillation, and $\omega_{0}=\beta c / R$ is the revolution frequency.

Including the space-charge force, the equation becomes

$$
\frac{d^{2} y_{p}}{d t^{2}}+\nu_{0 y}^{2} \omega_{0}^{2} y_{p}=\frac{F_{y}}{\gamma m_{p}}
$$

where $m_{p}$ is the rest mass of proton. The space-charge force may be divided into the three groups, namely incoherent direct space-charge force, incoherent image force, and coherent image force. Since the images forces are generally small compared to the direct space-charge force, we neglect image forces in our analysis. With this approximation, the equation becomes

$$
\frac{d^{2} y_{p}}{d t^{2}}+\left(\nu_{0 y}^{2} \omega_{0}^{2}-\frac{e^{2} \lambda}{\pi \epsilon_{0} b(a+b) \gamma^{3}}\right) y_{p}=0 .
$$

If we define the new tune $\nu_{y}^{2}=\left(\nu_{0 y}+\Delta \nu_{y}\right)^{2}$, we get for the incoherent tune shift

$$
\Delta \nu_{y}=-\frac{e^{2} \lambda}{2 \pi \epsilon_{0} \nu_{0 y} \omega_{0}^{2} b(a+b) m_{p} \gamma^{3}} .
$$

Introducing the classical electron/proton radius

$$
\left(r_{e}, r_{p}\right)=\frac{e^{2}}{4 \pi \epsilon_{0}\left(m_{e}, m_{p}\right) c^{2}}= \begin{cases}2.82 \times 10^{-15} \mathrm{~m} & \text { for electron } \\ 1.54 \times 10^{-18} \mathrm{~m} & \text { for proton }\end{cases}
$$

we may rewrite the tune-shift as

$$
\Delta \nu_{y}=-\frac{N_{p} R r_{p}}{\pi B \nu_{0 y} \beta^{2} \gamma}\left[\frac{1}{\gamma^{2} b(a+b)}\right] .
$$

For horizontal motion, we replace $y$ with $x$, and we interchange $a$ with $b$.

So far we have considered the motion of a proton inside the beam without considering the effect of trapped electron. Now we consider the space-charge effect of two species, i.e. electron and proton, we assume the following conditions are satisfied: 
1. Electrons are trapped inside the potential well of the circulating proton beam whether the beam is coasting or bunched.

2. Electrons are uniformly distributed in the longitudinal and transverse planes, and its cross section is the same as the circulating beam.

3. The longitudinal velocity of electrons is negligible. Thus the electrons are oscillating in transverse plane.

4. The motion of background ions does not have any considerable effects on either electron or proton motion.

With these assumptions, we first consider the effect on the tune shift of protons due to the electrons. This effect comes through the neutralization of proton beam, which results in the reduced space-charge force. If we define the neutralization

$$
\eta=\frac{\lambda_{e}}{\lambda_{p}}
$$

$\Delta \nu_{y}$ becomes

$$
\Delta \nu_{y}=-\frac{N_{p} R r_{p}}{\pi B \nu_{0 y} \beta^{2} \gamma}\left[\frac{1}{b(a+b)}\right]\left(\frac{1}{\gamma^{2}}-\eta\right) .
$$

Since $\eta$ is usually less than 0.1 , such an effect on tune shift may be neglected. For example, if we use $\nu_{0 x}=6.821$ and $\nu_{0 y}=5.731$ for the IPNS-Upgrade RCS, we find at the end of injection

$$
\Delta \nu_{x}=-\frac{0.06}{B}(0.5-\eta), \Delta \nu_{y}=-\frac{0.07}{B}(0.5-\eta) .
$$

The second effect is coupling of electron and proton motion. In this effect, the motion of protons is influenced by the space-charge force of electron. Including this effect, the equation of motion for the protons may be written as

$$
\frac{d^{2} y_{p}}{d t^{2}}+\nu_{y}^{2} \omega_{0}^{2} y_{p}=\frac{r_{p} \eta}{\gamma} \frac{2 c^{2} N_{p}}{\pi R b(a+b)}\left(Y_{e}-y_{p}\right)
$$

where $Y_{e}$ is the centroid of the electron beam.

Similarly, we obtain the equation of motion for the electrons

$$
\frac{d^{2} y_{e}}{d t^{2}}=\frac{r_{e}}{B} \frac{2 c^{2} N_{p}}{\pi R b(a+b)}\left(Y_{p}-y_{e}\right)
$$

where $Y_{p}$ is the centroid of the proton beam. 
Introducing the tunes of bounce oscillation of protons and electrons in the potential wells $Q_{p}$ and $Q_{e}$, respectively, we rewrite the equation of motion as

$$
\begin{aligned}
\frac{d^{2} y_{p}}{d t^{2}}+\nu_{y}^{2} \omega_{0}^{2} y_{p} & =Q_{p}^{2} \omega_{0}^{2}\left(Y_{e}-y_{p}\right), \\
\frac{d^{2} y_{e}}{d t^{2}} & =Q_{e}^{2} \omega_{0}^{2}\left(Y_{p}-y_{e}\right),
\end{aligned}
$$

where

$$
\begin{aligned}
Q_{p}^{2} & =\frac{r_{p} \eta}{\gamma} \frac{2 N_{p} R}{\pi b(a+b) \beta^{2}} \\
Q_{e}^{2} & =\frac{r_{e}}{B} \frac{2 N_{p} R}{\pi b(a+b) \beta^{2}} \\
\frac{Q_{p}^{2}}{Q_{e}^{2}} & =\frac{\eta B}{\gamma}\left(\frac{r_{p}}{r_{e}}\right) .
\end{aligned}
$$

The centroid of beam is defined by

$$
Y=\int y(p) f(p) d p
$$

where $f(p)$ is the normalized distribution function and $p$ is a variable that influences the betatron frequency. If we multiply $y$ in Eq. (17) by the distribution functions and integrate, we obtain:

$$
\begin{aligned}
\frac{d^{2} Y_{p}}{d t^{2}}+\nu_{y}^{2} \omega_{0}^{2} Y_{p} & =Q_{p}^{2} \omega_{0}^{2}\left(Y_{e}-Y_{p}\right) \\
\frac{d^{2} Y_{e}}{d t^{2}} & =Q_{e}^{2} \omega_{0}^{2}\left(Y_{p}-Y_{e}\right)
\end{aligned}
$$

where we assumed a uniform distribution.

We solve Eq. (18) assuming that the beam center oscillates in the following form:

$$
Y_{p}=A_{p} e^{j(\omega t-n \theta)}, Y_{e}=A_{e} e^{j \omega t} .
$$

Since the suggested dependence of $t$ and $\theta$ is a valid ansatz for a coasting beam, the following discussions are limited to the analysis of the coasting beam, i.e. $B=1$.

Substituting Eq. (19) into Eq. (18), we obtain the following dispersion equation: 


$$
f(x)=\left(Q_{e}^{2}-x^{2}\right)\left[\nu_{y}^{2}+Q_{p}^{2}-(n-x)^{2}\right]=Q_{e}^{2} Q_{p}^{2}=\epsilon,
$$

where $x=\omega / \omega_{0}$.

If we denote the roots, which satisfy $f(x)=\epsilon$, as $x_{1}, x_{2} x_{3} x_{4}$, we may order the roots in such a way that $x_{1}<x_{2}<x_{3}<x_{4}$. This is possible when the solutions all real and distinct, which represents a stable system. Instability arises when two solutions, say $x_{2}$ and $x_{3}$, become complex by pair. Threshold conditions which divide the stability region are when two solutions are double roots, i.e. $x_{2}=x_{3}$. At the threshold, $Q_{p}$ becomes

$$
Q_{p}^{t h}=\frac{\left(n-Q_{e}\right)^{2}-\nu_{y}^{2}}{2 \sqrt{Q_{e}\left(n-Q_{e}\right)}} .
$$

Once the threshold value of $Q_{p}$ is determined, then the corresponding neutralization can be determined by Eq. (17).

\section{Application}

The beam pulse from the linac is injected into the waiting bucket during the injection period. A total of 561 pulses from the linac will be accumulated during this period prior to the acceleration cycle. Each pulse is chopped occupying $75 \%$ of the ring circumference. However, due to RF action on the beam, the beam eventually occupies the $85 \%$ of the ring at the end of injection. This was shown by the extensive tracking studies [9], where the effects of space charge are included. The tracking studies also showed that the gap between the bunch is clean during the injection and acceleration cycles without losing any particles.

However, the longitudinal beam distribution in the waiting bucket during injection is sensitive to both chopper efficiency and injection mismatch from the linac. Thus we apply theory to the injection period.

Substituting RCS parameters, we find that $Q_{e}=46.47$. Since the machine tune is $\nu_{y}=5.731$, the most dangerous mode $n$ is 53 . Thus, the threshold value ${ }^{2}$ is $Q_{p}=0.2817$. By using Eq. (17) we found that the required neutralization is $\eta \simeq 0.1$ at the injection.

Since the parameters that appeared in the dispersion equation are functions of beam size, we investigate such a dependence: results are shown in Fig. 2 . In the calculation, we assumed a round beam with uniform density. By choosing the tune in a wide range, it covered the threshold conditions in both horizontal and vertical

\footnotetext{
${ }^{2}$ Figure 1 shows the curve $f(x)=\epsilon$ for several values of $Q_{p}$. It can be seen that as $Q_{p}$ decreases, $f_{\max }$ increases and $\epsilon$ decreases; the two values become equal when $Q_{p}=Q_{p}^{\text {th }}$.
} 
directions. In the interesting range of tunes, namely $5.6<\nu_{y}<5.75$ and $6.7<\nu_{x}<$ 6.85 , the minimum required neutralization is about 0.05 .

Neutralization from the residual gas was shown to satisfy the following empirical formula [10],

$$
\eta=0.842 P(n \text { Torr }) t(s e c),
$$

where $P$ is $N_{2}$ baseline pressure and $t$ is time. In deriving the formula, the variation of ionization cross section with respect to the kinetic energy was taken into account. Thus, the formula is valid for one cycle of RCS operation. The vacuum pressure must be less than $10^{-7}$ Torr for a $t=0.5$ msec injection time. Since the design value is $10^{-8}$ Torr, the electrons will not be accumulated to the threshold value. Thus, even if the gap is filled, we may not observe the e-p instability caused by the beam neutralization from the background molecules.

\section{Conclusion}

In this report, we investigated the possibility of e-p instability in the IPNS-Upgrade RCS caused by beam neutralization from background molecules. Coasting beam theory was used to estimate the threshold neutralization for the onset of e-p instability. The results show that the onset of instability requires beam neutralization of 5 percent. At the end of injection, the actual neutralization is found to be a factor of ten smaller than the threshold value. Thus, we don't expect to observe the e-p instability in the RCS during the injection period.

After injection, the beam is further bunched leaving a clean. wide gap between bunches. This will prevent the accumulation of electrons in the circulating proton beam.

What is not covered in this report is the effects of the localized electron cloud. The source of these electrons are the stripping foil and any collimators installed in the ring in order to localize the beam loss. This is potentially dangerous, because the circulating bunch could interact with the electron cloud during every turn of the injection period. Thus, we recommend clearing electrodes be installed around the foil and the collimators to circumvent this potentially dangerous situation.

The question of the bunched beam distribution on instability and the effect of the localized electron cloud require further refinement of theory and simulation technique. These subject will be treated in a separate report. 


\section{References .}

[1] E. Keil and B. Zotter, "Landau-damping of coupled electron-proton oscillations," CERN-ISR-TH/71-58, 1971.

[2] C. Planner, "Reaching $200 \mu \mathrm{A}$ on ISIS," Proc. 12th ICANS Workshop Abingdon UK, Vol. II, May, 1993.

[3] D. Neuffer et al., "Observation of a fast transverse instability in the PSR," NIM A321, pp. 1-12, 1992.

[4] Y. Baconnier, "Neutralization of Accelerator Beams by Ionization of the Residual Gas," Proc. CERN Accelerator School, CERN 85-19, Vol. 1, pp. 267-300, 1985.

[5] D. G. Koshkarev and P. R. Zenkevich, "Resonance of Coupled Transverse Oscillations in Two Circular Beams," Part. Accel., Vol. 3, pp. 1-9, 1972.

[6] L. J. Laslett and A. M. Sessler, "Transverse Two-Stream Instability in the Presence of Strong Species-Species and Image Forces," NIM 121, pp. 517-524, 1974.

[7] L. C. Teng, "Transverse Space Charge Effects," ANLAD-59, February, 1960.

[8] F. Lapique and F. Piuz, "Simulation of the measurement by primary cluster counting of the energy lost by a relativistic ionizing particle in Argon," NIM 175, pp. 297-318, 1980.

[9] E. Lessner, private communication, January, 1995.

[10] Y.C. Chae, "Calculation of Beam Neutralization in the IPNS-Upgrade RCS," Argonne Report NSA-95-1, January, 1995. 

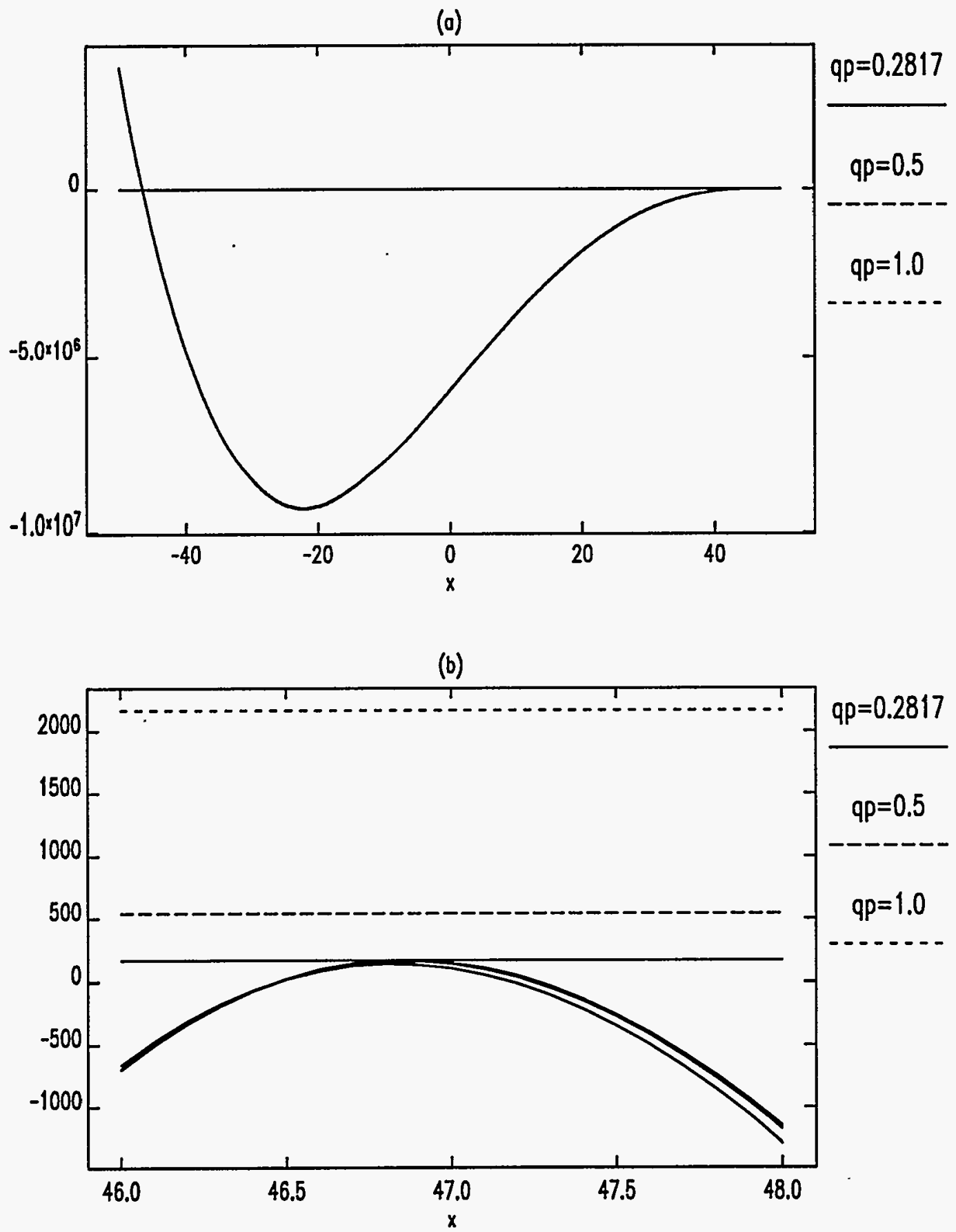

Figure 1: Graphical representation of dispersion relation. 
Tune vs. Neutralization

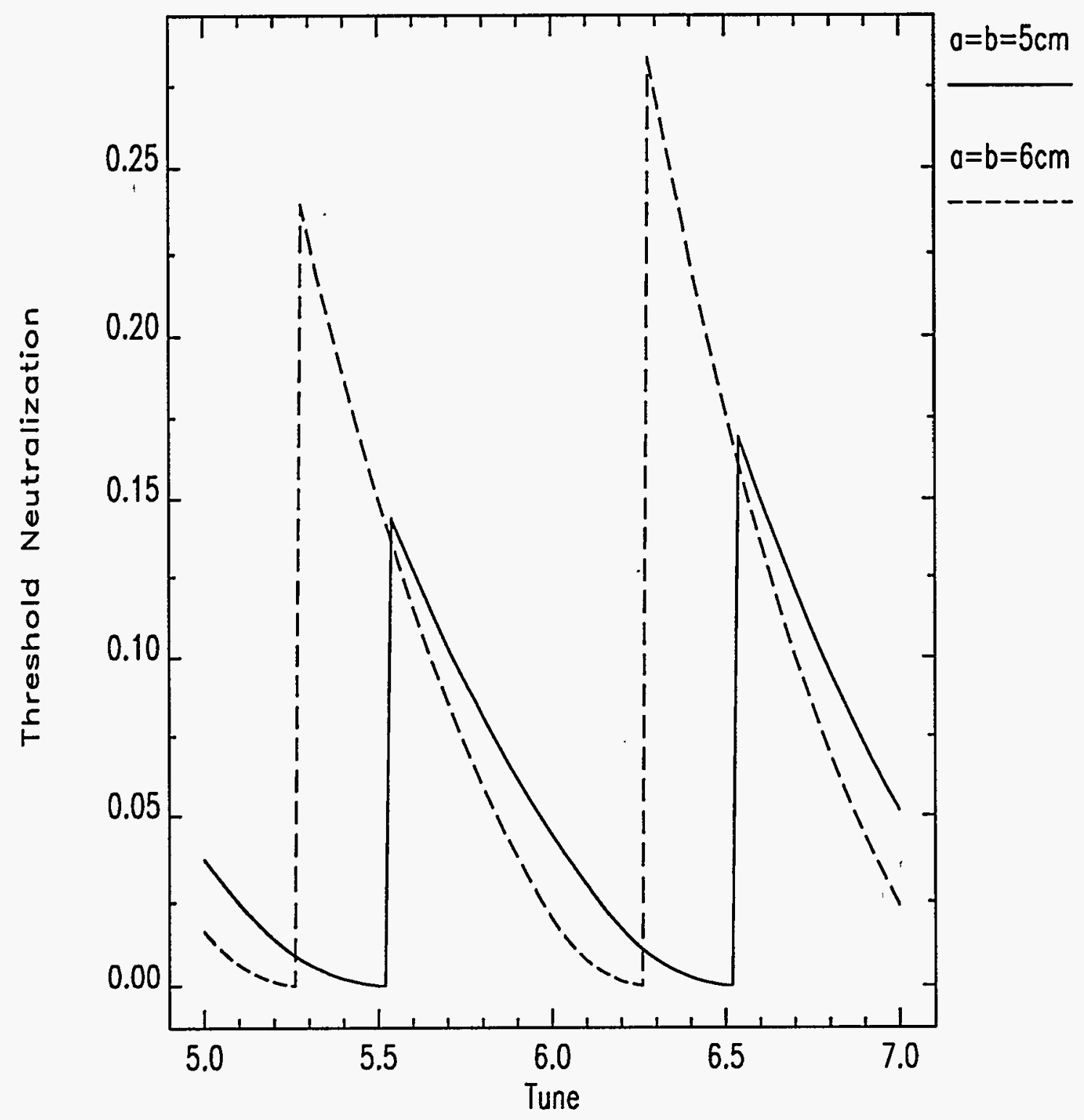

Figure 2: Threshold neutralization as functions of tune for the different beam sizes. 